\title{
The new laparoscopic uterine sling suspension procedure: first year follow-up data
}

\author{
Arvind Vashisht • Rohna Kearney • Alfred Cutner
}

Received: 7 December 2010 / Accepted: 14 December 2010/Published online: 5 January 2011

(C) Springer-Verlag 2010

\begin{abstract}
Ideally, any uterine conservation procedure for the treatment of prolapse should be effective, and yet, not significantly jeopardise subsequent surgery should a hysterectomy become a necessity in the future. We present our 1 -year data on the new laparoscopic uterine sling suspension procedure. We have found significant anatomical improvements in points $\mathrm{C}$ and $\mathrm{D}$ on the pelvic organ prolapse quantification score, and improvements in vaginal, sexual and quality of life scores. We conclude that this technique is a promising new surgical method for treating uterine prolapse.
\end{abstract}

Keywords Laparoscopy · Uterine prolapse · Fertility sparing $\cdot$ POP-Q

\section{Background}

Uterine prolapse is a common cause for referral to a urogynaecology clinic. A substantial number of these women request a surgical solution for their problem. These women are often well treated with a hysterectomy coupled with some form of vault suspension procedure with the aim of preventing further central compartment

\footnotetext{
A. Vashisht $(\bowtie) \cdot$ A. Cutner

Department of Obstetrics and Gynaecology,

University College Hospital,

250 Euston Road,

London NW1 2PG, UK

e-mail: lisa@gynaecology.co

R. Kearney

Department of Obstetrics and Gynaecology,

Addenbrooke's Hospital,

Cambridge, UK
}

prolapse. Within the women requesting a surgical solution, those women keen for uterine preservation, represent a difficult management group. A variety of different vaginal, abdominal and laparoscopic approaches have been tried to correct prolapse, and yet, conserve the uterus, with varying degrees of success $[1,2]$. To appropriately observe change in vaginal anatomical parameters, it is important to use a validated pre- and postoperative prolapse scoring system. In addition, the yardsticks for treatment success need to encompass patient symptoms and satisfaction, as these are the ultimate arbiters of any prolapse management technique.

\section{Methods}

All women, having surgery were notified that the procedure was a new procedure with consequently no follow-up data, had been approved by the local hospital new procedures of the Clinical Governance Committee, and that it was registered with the National Institute for Clinical Excellence. The surgical technique has been previously described and published in full [3]. Laparoscopic access is gained into the pelvis. The uterosacral ligament remnants are identified and bilateral ureterolysis are performed. A Mersilene tape is introduced and passed through the uterus at the cervicoisthmic junction, through both remnant ligaments, tunnelled under the peritoneum, and finally, attached bilaterally to the sacral promontory. In essence, the procedure serves to create two new modified uterosacral ligaments.

Women were followed up at 3,6 and 12 months. They filled out a preoperative and postoperative vaginal symptom score questionnaire. The International Consultation on Incontinence Questionnaire (ICIQ) vaginal symptoms questionnaire was used as it is a validated tool, particularly 
useful in pelvic organ prolapse patients, encompassing domains specific to vaginal and sexual symptoms and quality of life [4]. All women had a pelvic organ prolapse quantification score (POP-Q) [5] recorded preoperatively and at each follow-up visit.

\section{Findings}

Data are presented for the ten women who have completed 1-year follow-up. The median age was 37.3 years (interquartile range, 33.9-44.7). The median hospital stay was 2 days (2-3). Median operative time was $125 \mathrm{~min}$ (98-141). Concomitant anterior compartment surgery was carried out in five women. Two had a paravaginal repair, two had a colposuspension and paravaginal repair, and one had a colposuspension.

The median change in POP-Q measurement over 1 year is shown in Fig. 1 (median, quartiles and range are shown). Differences between the pre- and postoperative measurements in the same individual were compared using Wilcoxon matched pairs test. Significant differences were seen in the measurements at $\mathrm{Aa}(p=0.015), \mathrm{Ba}(p=0.009)$, $\mathrm{C}(p=0.005)$ and $\mathrm{D}(p=0.005)$, reflecting the improvements in anterior and apical support. The other components of the POP-Q score were not significantly changed.

Significant improvements were seen in all three domains of the ICIQ 1 year following surgery compared to preoperative: vaginal symptoms score $(p=0.01)$, sexual matters score $(p=0.005)$ and quality of life score $(p=$ 0.012 ). The procedure was successfully completed in all patients, with no immediate perioperative complications, apart from one woman who developed surgical emphysema, related to a combination of pneumoperitoneum and reverse Trendelenburg. This resolved satisfactorily $6 \mathrm{~h}$ following surgery. Two women failed their trial of voids on

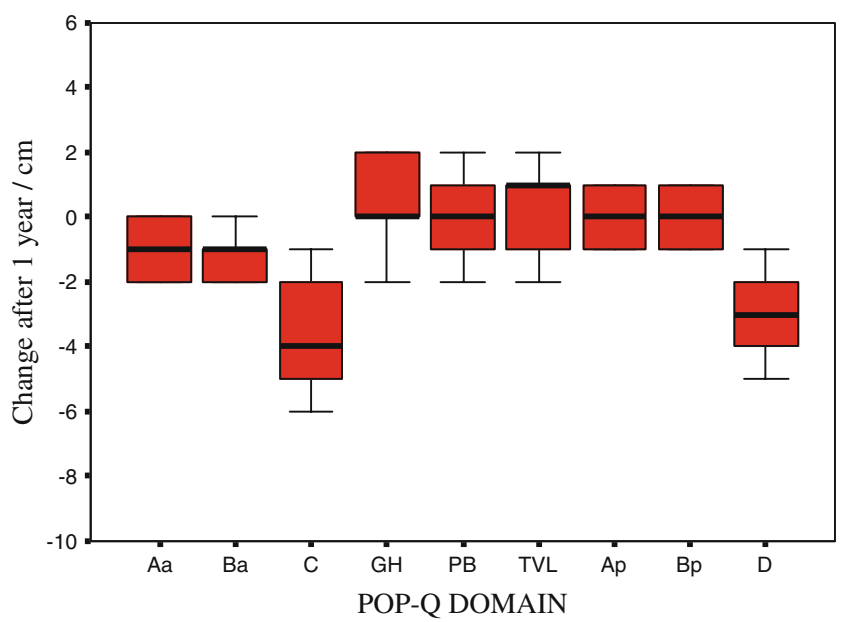

Fig. 1 Change in each POP-Q domain after 1 year the second postoperative day and needed to be catheterised for a further week

\section{Conclusions}

Laparoscopic uterine sling suspension is a novel way of maintaining uterine support for those women specifically requesting for uterine conservation and surgical management of uterine prolapse (Fig. 2). For many women, uterine conservation is an absolute necessity to retain future reproductive potential. For others, it remains a matter of personal choice. Newer techniques are being introduced that respond to patient's desires for such conservative surgery and their desire for minimally invasive techniques. Before widespread implementation, it is imperative that new procedures are subject to scrutiny, with a proven safety profile and evidence of clinical effectiveness.

We have seen no incidence of significant peri- or postoperative complications. In addition, we have observed significant improvements in vaginal symptoms of prolapse, with consequent improvements in sexual function and quality of life. As expected, anatomically, the improvements are noted in the central indices of the POP-Q measurement (the $\mathrm{C}$ and $\mathrm{D}$ measurements). We also found significant improvements in the anterior compartment measurements after 1 year. These results are due to the fact that half the women had either a concomitant colposuspension or paravaginal repair or both.

The benefits of our laparoscopic uterine sling are the enhanced visualisation of the pelvis and relevant anatomy that laparoscopy affords. Aside from the initial entry into the abdomen, all surgical steps are undertaken under direct vision, minimising the risks of inadvertent ureteric or bowel damage. The inpatient stay is modest and the patients cite an early return to normal activities.

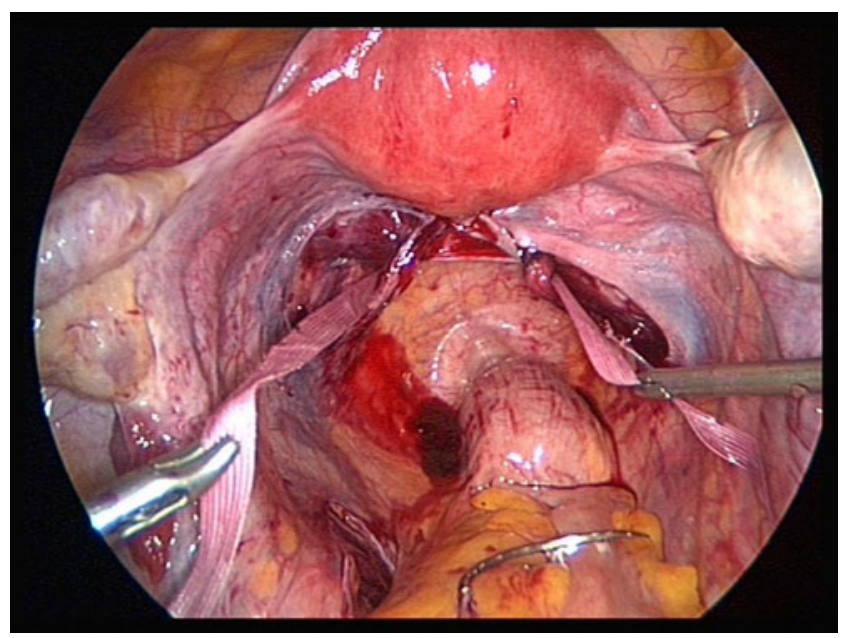

Fig. 2 Laparoscopic uterine sling suspension procedure 
Prolapse surgery has seen a rapid introduction of abdominal and vaginal mesh kits. Some of these devices have led to unacceptably high dyspareunia rates, often a result of palpable or eroded mesh [6]. As there is no formal opening of the vagina and the mesh is inserted above the level of posterior fornix, the likelihood of any of the commonly reported vaginal erosion associated with vaginal meshes is likely to be low. The mesh is lateralised by passing it through the uterosacral ligaments and buried beneath the peritoneum, so the risk of bowel complication is likely to be low.

One of the perceived problems with other vaginal and laparoscopic mesh uterine suspension procedures is that should a women need a subsequent hysterectomy in the future, the significant mesh attachments would make the operation hazardous. We anticipate that should any of the women in our series require subsequent hysterectomy, the procedure would begin by two simple cuts to divide the tape (i.e., the recreated uterosacral ligaments), and then the uterus could be removed in a routine fashion.

The robustness and clinical usefulness of any prolapse procedure needs to be observed over many years. We have previously demonstrated that the procedure is well tolerated and without immediate significant complications. We have now been able to demonstrate the effectiveness of the procedure over a period of 1 year, both in terms of objective anatomical measurements and in terms of patient-reported symptoms.

Conflicts of interest None.

\section{References}

1. Carey MP, Slack MC (1994) Transvaginal sacrospinous colpopexy for vault and marked uterovaginal prolapse. Br J Obstet Gynaecol 101(6):536-540

2. Leron E, Stanton SL (2001) Sacrohysteropexy with synthetic mesh for the management of uterovaginal prolapse. BJOG 108(6):629-633

3. Cutner A, Kearney R, Vashisht A (2007) Laparoscopic uterine sling suspension: a new technique of uterine suspension in women desiring surgical management of uterine prolapse with uterine conservation. BJOG 114(9):1159-1162

4. Price N, Jackson SR, Avery K, Brookes ST, Abrams P (2006) Development and psychometric evaluation of the ICIQ vaginal symptoms questionnaire: the ICIQ-VS. BJOG 113(6):700-712

5. Bump RC, Mattiasson A, Bo K, Brubaker LP, DeLancey JO, Klarskov P et al (1996) The standardization of terminology of female pelvic organ prolapse and pelvic floor dysfunction. Am J Obstet Gynecol 175(1):10-17

6. Baessler K, Hewson AD, Tunn R, Schuessler B, Maher CF (2005) Severe mesh complications following intravaginal slingplasty. Obstet Gynecol 106(4):713-716 